Universidade Tecnológica Federal do Paraná - UTFPR

Campus Ponta Grossa - Paraná - Brasil

ISSN: 1981-3686/v. 01, n. 02: p. $37-47$
Revista Brasileira de Tecnologia

Agroindustrial

\title{
COMPOSIÇÃO CENTESIMAL E TEOR DE MINERAIS DE DIETAS ENTERAIS ARTESANAIS
}

\section{CENTESIMAL COMPOSITION AND MINERAL CONTENT OF HOMEMADE ENTERAL DIETS}

\author{
Maria Carolina Von Atzingen ${ }^{1}$; Maria Lima Garbelotti ${ }^{2}$; Roberta Faria Camilo Araújo ${ }^{3}$; Rosana Manólio \\ Soares ${ }^{4}$; Maria Elisabeth Machado Pinto e Silva ${ }^{5}$ \\ ${ }^{1}$ University of São Paulo - FSPUSP - São Paulo - Brasil. mcva@usp.br \\ ${ }^{2}$ Instituto Adolfo Lutz - IAL - São Paulo - Brasil. mgarbelo@ial.sp.gov.br \\ ${ }^{3}$ Federal University of São Paulo - UNIFESP - São Paulo - Brasil. omsamancio.dped@epm.br \\ ${ }^{4}$ University of São Paulo - FSPUSP - São Paulo - Brasil. rosanaso@usp.br \\ ${ }^{5}$ University of São Paulo- FSPUSP - São Paulo - Brasil. mmachado@usp.br
}

\begin{abstract}
Resumo
O objetivo do presente trabalho foi determinar a composição centesimal de dietas enterais artesanais à base de hidrolisado protéico de carne. Métodos. A obtenção do hidrolisado protéico de carne foi a descrita por PINTO e SILVA et al, 1999 . Foram analisadas três fórmulas com hidrolisado de carne (bovina, frango ou peru) acrescidas de arroz, cenoura, beterraba, amido de milho, glicose de milho e óleo de soja. A composição centesimal foi determinada pela metodologia preconizada pelo INSTITUTO ADOLFO LUTZ, 1985. A análise de colesterol foi obtida por HPLC. Os ácidos graxos foram separados por cromatografia gasosa de alta resolução. Os valores de fibra alimentar total pela metodologia apresentada por PROSKY et al, 1988. Os minerais foram determinados por espectrofotometria de Absorção Atômica em aparelho Perkin Elmer modelo 5.100. Resultados. As fórmulas foram classificadas como hipocalóricas. O teor protéico das fórmulas variou entre 2.09 e 2.52g/100g, lipídeos entre 1.04 e $1.32 \mathrm{~g} / 100 \mathrm{~g}$, carboidratos 9.32 e $9.67 \mathrm{~g} / 100 \mathrm{~g}$, com $8 \mathrm{~g} / \mathrm{L}$ de fibras. O colesterol variou entre $14.32 \mathrm{mg} / 100 \mathrm{~g}$ (frango) e $37.59 \mathrm{mg} / 100 \mathrm{~g}$ (bovino). As três fórmulas desenvolvidas apresentam maior concentração de ácidos graxos monoinsaturados, com prevalência do oléico. Em relação aos minerais, os valores de cobre variaram entre $0.07 \mathrm{mg} / 100 \mathrm{~g}$ e $0.06 \mathrm{mg} / 100 \mathrm{~g}$, zinco $0.28 \mathrm{mg} / 100 \mathrm{~g}$ e $0.62 \mathrm{mg} / 100 \mathrm{~g}$, ferro 0.47 $\mathrm{mg} / 100 \mathrm{~g}$ e $0.63 \mathrm{mg} / 100 \mathrm{~g}$, magnésio $8.08 \mathrm{mg} / 100 \mathrm{~g}$ e $8.58 \mathrm{mg} / 100 \mathrm{~g}$ e cálcio $1.34 \mathrm{mg} / 100 \mathrm{~g}$ e 1.40 $\mathrm{mg} / 100 \mathrm{~g}$. As fórmulas apresentaram teor protéico adequado com predominância de ácidos graxos monoinsaturados, sendo indicadas a pacientes que requerem suplementação nutricional.
\end{abstract}

Palavras-chave: nutrição enteral, composição centesimal, minerais.

\section{Introdução}

A nutrição enteral é caracterizada pelo uso de suplementação oral e/ou enteral, em casos de impossibilidade ou restrição de utilização da via oral, como forma de prover a totalidade ou parte dos nutrientes necessários (BAXTER et al, 2001, NISIM \& ALLIMS, 2005). 
Tem-se verificado um crescente aumento do uso de nutrição enteral em terapia domiciliar, visando promover a manutenção e/ou melhora do estado nutricional (EPLEY, 1999, ARZUJO \& MENEZES, 2005), com considerável prevalência do uso de formulados artesanais (DOMENE \& GALEAZZI, 1997, YAMAUTI et al, 1999, MORAIS \& VIEIRA, 2005).

Estudo realizado por DOMENE \& GALEAZZI, 1997, em instituições hospitalares de Campinas, constatou que há maior prevalência do uso de formulados artesanais para pacientes no domicílio e dos industrializados para pacientes internados.

Um grande número de formulações comerciais com diversas aplicações está disponível no mercado (PREETIKA et al, 2004), entretanto, são de alto custo e, quando administradas por longo período, leva os pacientes a utilizar fórmulas caseiras (SULLIVAN et al, 2001).

Em países da América do Norte e Europa, as dietas enterais artesanais foram praticamente eliminadas, entretanto continuam a ser utilizadas em outras regiões do mundo por razões econômicas e culturais, especialmente naquelas em desenvolvimento (SILVA, 2002, HEBUTERNE et al. 2003, MONTEJO et al, 2001). Tais fórmulas promovem individualização da dieta nos casos em que as necessidades de alguns pacientes não são satisfeitas por fórmulas padrão, sendo necessário o monitoramento do valor nutricional desse tipo de dieta para obter maior segurança na ingestão de nutrientes (ARAUJO \& MENEZES, 2005).

Há escassez de dados nacionais referentes à análise da composição centesimal de dietas enterais artesanais e tais informações são importantes principalmente para o estabelecimento de parâmetros de avaliação da qualidade nutricional de dietas (MONTEJO et al, 2001). O objetivo deste trabalho foi determinar a composição centesimal de dietas enterais artesanais à base de hidrolisado protéico de carne.

\section{Material e Métodos}

\section{1. Dietas enterais artesanais}

Para a obtenção do hidrolisado bovino foi utilizado o corte comercializado como "patinho", e para os hidrolisados de frango e peru, o peito. A fonte de enzima proteolítica empregada foi o suco de abacaxi (Ananas comosus) in natura. Iguais proporções de carne e suco foram homogeneizados em liquidificador (Pic-Liq Arno®, São Paulo, Brasil) e mantidos em banho-maria $\left(60^{\circ} \mathrm{C}\right)$ por 30 minutos em fogão doméstico, seguido de fervura por 5 minutos, conforme Pinto e Silva et al, 1999, adaptado por Atzingen, 2005.

Foram analisadas três fórmulas com hidrolisado de carne bovina, frango ou peru $(200 \mathrm{~g})$ acrescidas de arroz cozido $(200 \mathrm{~g})$, cenoura $(180 \mathrm{~g})$, beterraba $(180 \mathrm{~g})$, amido de milho $(50 \mathrm{~g})$, glicose 
de milho (100g) e óleo de soja (40g). Os legumes cozidos em panela de pressão (12psi, cap. 4L, Clock ${ }^{\circledR}$, Brasil) foram homogeneizados com os demais ingredientes em liquidificador (Pic-Liq Arno ${ }^{\circledR}$, São Paulo, Brasil doméstico), acrescentando-se a água do cozimento até completar 1 litro. Em seguida, a dieta foi levada ao fogo baixo por 5 minutos e passada em peneira de malha fina (tela de nylon plana, abertura de $1 \mathrm{~mm}$ de diâmetro).

Os alimentos foram pesados em balança analítica (Mettler Toledo® PB 3002 precisão $0,1 \mathrm{mg})$.

Os alimentos foram adquiridos em supermercados da cidade de São Paulo.

\subsection{Composição centesimal}

Umidade, cinzas, proteínas, lipídeos e carboidratos foram obtidos pela metodologia preconizada pelo Instituto Adolfo Lutz, 1985.

Colesterol

Os lípides foram extraídos em coluna seca (MARMER \& MAXWELL, 1981) utilizando-se como solventes diclorometano-metanol na proporção 9:1. Foi feita saponificação prévia das amostras e extração do colesterol por hexano de acordo com BOHAC et al, 1988. A análise de colesterol foi obtida por CLAE da marca TSP (THERMO SEPARATION PRODUCTS).

Utilizou-se uma coluna de CN (fase intermediária) e os solventes hexano-isopropanol. A fase móvel foi uma mistura de 97:3 de hexano-isopropanol, com fluxo de 1mL/min. Foram injetados $20 \mu \mathrm{L}$ da amostra (CHEN \& CHEN, 1994, VICENTE, 2003). A detecção do colesterol se deu no comprimento de onda de máxima absorção (206nm) em detector UV/vis.

Ácidos graxos

Os ácidos graxos no extrato foram saponificados previamente e esterificados com uso de BF3 (MORRISON \& SMITH, 1964). Posteriormente foram separados por cromatografia gasosa de alta resolução em cromatógrafo à gás marca Chrompack modelo CP9002 equipado com injetor tipo "split/splitless" e coluna capilar de sílica fundida "CP-SIL 88" (50m de comprimento x 0,25mm de diâmetro interno), própria para essa análise. A razão de divisão utilizada é 1:60, pressão do gás hidrogênio 16psi. Temperatura de injeção: $270^{\circ} \mathrm{C}$, detector FID $300^{\circ} \mathrm{C}$. Forno inicial $100^{\circ} \mathrm{C}$ até $240^{\circ} \mathrm{C}$ a taxa de $5^{\circ} \mathrm{C} / \mathrm{min}$.

Foi utilizado como padrão lípide Standart SIGMA ${ }^{\circledR}$ - Fatty acids methyl ester mixtures $n^{\circ}$ 189-19. 
Fibras

Os valores de fibra alimentar total foram obtidos a partir da metodologia utilizada por

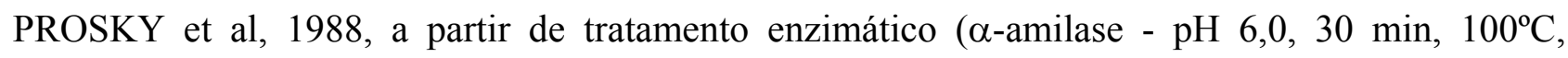
protease - $\mathrm{pH} 7,5,30 \mathrm{~min}, 60^{\circ} \mathrm{C} \mathrm{e}$, amiloglicosidade - $\mathrm{pH} 4,75,30 \mathrm{~min}, 60^{\circ} \mathrm{C}$ ).

Minerais

Foi realizada digestão líquida prévia com $\mathrm{HNO}_{3}$ (ácido nítrico) e $\mathrm{HCLO}_{4}$ (ácido perclórico) e os minerais determinados por espectrofotometria de Absorção Atômica em aparelho Perkin Elmer modelo 5.100 nas seguintes condições: Comprimento de onda: $\mathrm{Cu}=324,8 \mathrm{~nm} ; \mathrm{Mg}=285,2 \mathrm{~nm} ; \mathrm{Zn}$ $=213,9 \mathrm{~nm} ; \mathrm{Fe}=248,3 \mathrm{~nm} ; \mathrm{Ca}=422,7 \mathrm{~nm}$; fenda $0,7 \mathrm{~nm}$; chama ar/acetileno; energia: $\mathrm{Cu}=71 ; \mathrm{Mg}$

$=69 ; \mathrm{Zn}=65 ; \mathrm{Fe}=60 ; \mathrm{Ca}=67$; leitura em duplicata de 10 amostras de cada tipo de dieta com tempo de integração de 2 segundos (MARKS et al, 1971). Os resultados foram expressos em $\mu \mathrm{g}$ metal/ 100g dieta e desvio padrão.

\subsection{Distribuição de nutrientes e densidade energética}

A distribuição de macronutrientes e o valor calórico total foram obtidos a partir da composição centesimal utilizando-se os fatores de conversão de Atwater. A densidade energética foi calculada de acordo com o fornecimento de calorias por $\mathrm{mL}$.

\subsection{Análise estatística}

Os resultados foram apresentados como médias e desvio-padrão.

A análise estatística dos resultados obtidos na composição centesimal foi realizada a partir da média dos valores obtidos em triplicata $(n=9)$. 


\section{Resultados}

Tabela 1- Composição centesimal e minerais (média \pm desvio-padrão) das dietas elaboradas com hidrolisado bovino (FHB), de frango (FHF) e de peru (FHP)

\begin{tabular}{cccc}
\hline & FHB & FHF & FHP \\
\hline Umidade $(\mathrm{g} / 100 \mathrm{~g})$ & $85,8 \pm 0,38$ & $85,6 \pm 0,30$ & $85,3 \pm 0,38$ \\
Cinzas $(\mathrm{g} / 100 \mathrm{~g})$ & $0,27 \pm 0,01$ & $0,27 \pm 0,02$ & $0,29 \pm 0,04$ \\
Proteína $(\mathrm{g} / 100 \mathrm{~g})$ & $2,09 \pm 0,18$ & $2,37 \pm 0,29$ & $2,52 \pm 0,10$ \\
Lipídeos $(\mathrm{g} / 100 \mathrm{~g})$ & $1,32 \pm 0,30$ & $1,27 \pm 0,00$ & $1,04 \pm 0,07$ \\
Colesterol $(\mathrm{mg} / 100 \mathrm{~g})$ & $35,9 \pm 2,21$ & $16,24 \pm 1,43$ & $28,65 \pm 0,97$ \\
Carboidrato $(\mathrm{g} / 100 \mathrm{~g})$ & $9,38 \pm 0,53$ & $9,32 \pm 0,23$ & $9,67 \pm 0,65$ \\
Fibras $(\mathrm{g} / 100 \mathrm{~g})$ & $0,98 \pm 0,03$ & $0,88 \pm 0,05$ & $0,82 \pm 0,03$ \\
Cobre $(\mathrm{mg} / 100 \mathrm{~g})$ & $0,07 \pm 0,00$ & $0,06 \pm 0,01$ & $0,06 \pm 0,01$ \\
Zinco $(\mathrm{mg} / 100 \mathrm{~g})$ & $0,62 \pm 0,05$ & $0,28 \pm 0,03$ & $0,29 \pm 0,02$ \\
Ferro $(\mathrm{mg} / 100 \mathrm{~g})$ & $0,63 \pm 0,06$ & $0,59 \pm 0,09$ & $0,47 \pm 0,02$ \\
Magnésio $(\mathrm{mg} / 100 \mathrm{~g})$ & $8,58 \pm 0,65$ & $8,76 \pm 0,66$ & $8,08 \pm 0,58$ \\
Cálcio $(\mathrm{mg} / 100 \mathrm{~g})$ & $1,34 \pm 0,11$ & $1,4 \pm 0,09$ & $1,41 \pm 0,13$
\end{tabular}

Base úmida, $\mathrm{n}=9$

As três dietas desenvolvidas apresentaram valor protéico superior $(2,09$ a $2,52 \mathrm{~g} / 100 \mathrm{~g})$ ao apresentado por MITNE et al, 2001, quando avaliou dietas artesanais com carne bovina (0,5 e 1,4g/100g), conforme observado na tabela 1. Em relação ao teor de lipídeos foram observados valores inferiores $(1,04$ a $1,32 \mathrm{~g} / 100 \mathrm{~g})$ e os carboidratos $(9,32$ a $9,67 \mathrm{~g} / 100 \mathrm{~g})$ estão dentro da variação verificada no estudo de MITNE (de 7,6 a 18g/100g).

Quanto ao fornecimento de fibras, as três dietas apresentam pelo menos $8 \mathrm{~g} / \mathrm{L}$, estando de acordo com o preconizado para dietas enterais, de 4 a 20g/L (CAMPBELL et al, 1997, BAXTER et al, 2001).

Os valores de colesterol variaram entre $32,65 \mathrm{mg} / 100 \mathrm{~g}$ e $37,59 \mathrm{mg} / 100 \mathrm{~g}$ para a fórmula com hidrolisado bovino, entre $14,32 \mathrm{mg} / 100 \mathrm{~g}$ e $17,7 \mathrm{mg} / 100 \mathrm{~g}$ com a de frango e entre $26,6 \mathrm{mg} / 100 \mathrm{~g}$ e $29,68 \mathrm{mg} / 100 \mathrm{~g}$ com a de peru. A fórmula com hidrolisado bovino é a que apresenta maior teor de colesterol, considerando-se que a carne bovina apresenta maior teor deste nutriente $(126 \mathrm{mg} / 100 \mathrm{~g})$ em relação às carnes de frango $(89 \mathrm{mg} / 100 \mathrm{~g})$ e de peru $(91 \mathrm{mg} / 100 \mathrm{~g})(\mathrm{NEPA}, 2007)$.

Considerando-se o consumo do volume total das dietas (2 litros), as fórmulas com hidrolisado bovino, frango e peru, fornecem respectivamente 1,4mg; 1,2 e 1,2 mg de cobre, o que corresponde a 1,5\% das DRI (INSTITUTE OF MEDICINE, 2000), para um indivíduo padrão. Quanto ao zinco, a fórmula com hidrolisado bovino corresponde à 155\% das recomendações, e as com frango e peru atingem pelo menos $70 \%$ do preconizado.

Para o ferro, as fórmulas com hidrolisado bovino, frango e peru contém, respectivamente 70\%, 65\% e 52\% das DRI (INSTITUTE OF MEDICINE, 2000). O fornecimento de magnésio é de no mínimo $50 \%$ das recomendações para as três fórmulas e o de cálcio é inferior a $5 \%$. 
Verifica-se um déficit no teor de minerais das fórmulas o que é indicativo de um monitoramento do paciente para verificar a necessidade de suplementação.

Quanto ao teor de ácidos graxos, verificou-se que as três fórmulas desenvolvidas apresentam maior concentração de ácidos graxos monoinsaturados, com prevalência do oléico, perfil benéfico, considerando-se a associação do consumo de gorduras saturadas com o desenvolvimento de doenças cardiovasculares, sendo a fórmula com hidrolisado bovino a de maior teor de saturados, conforme observado na tabela 2.

Tabela 2 - Teor (\%) de ácidos graxos das fórmulas enterais artesanais

\begin{tabular}{cccc}
\hline & FHB & FHF & FHP \\
\hline Ácido mirístico & 0.13 & 0 & 0 \\
Palmítico & 11.5 & 11.0 & 1.48 \\
Palmitoleico & 0.14 & 0.2 & 0 \\
Heptadecanóico & 0.10 & 0 & 0 \\
Heptadecenóico & 4.56 & 3.7 & 0.29 \\
Esteárico & 24.64 & 23.58 & 0.39 \\
Elaídico & 0.2 & 0.2 & 52.39 \\
Oleico & 50.42 & 53.46 & 0.42 \\
Linoleico & 0.41 & 0.3 & 0.27 \\
Gama linolênico & 0.26 & 0.3 & 5.55 \\
Araquidico & 5.15 & 6.00 & 0.63 \\
Alfa linolênico & 0.07 & 0 & 0 \\
Eicosenóico & 0.03 & 0.45 & 0.51 \\
Eucosadienóico & 0.5 & 0 & 0 \\
Behênico & 0.36 & 0 & 0 \\
Omega 3 eicosatrienóico & 0.42 & 0 & 0 \\
Omega 6 eicosatrienóico & 0.29 & 0 & 0 \\
Araquidônico & 0.30 & 0 & 0.05 \\
Capróico & 0 & 0 & 0.12 \\
Caprílico & 0 & 0 & 0.12 \\
Cáprico & 0 & 0 & 0.12 \\
Láurico & 0 & 0 & 11.2 \\
Mirístico & 0 & 0 & 0.07 \\
Miristoléico & 0 & 0 & 0.19 \\
Pentadecanoico & 0 & 0 & 4.07 \\
Pentadecenoico & 0 & 0 & 0.40 \\
Linolenelaidico & 0 & &
\end{tabular}

As fórmulas foram classificadas como hipocalóricas, segundo a categorização das fórmulas enterais pela sua densidade energética, conforme observado na tabela 3. Quanto à distribuição de nutrientes, as três fórmulas elaboradas apresentam-se próximo do estabelecido para dieta enteral, em relação à distribuição de proteínas, ou seja, de 14 a 20\%, no entanto, houve um desequilíbrio dos outros nutrientes, considerando-se a recomendação de 30 a $35 \%$ do valor calórico total proveniente de lipídeos e de 40 a 60\% de carboidratos (BAXTER et al, 2001). Esta distribuição deverá ser levada em consideração na sua aplicação, fazendo as correções necessárias, quanto à proporção de ingredientes e líquidos empregada. 
Tabela 3 - Distribuição das dietas elaboradas segundo distribuição de macronutrientes (\%VCT) e densidade energética

\begin{tabular}{ccccc}
\hline Tipo de fórmula & \multicolumn{3}{c}{$\begin{array}{c}\text { Distribuição de } \\
\text { macronutrientes }(\% \mathrm{VCT})\end{array}$} & $\begin{array}{c}\text { Densidade energética } \\
(\mathrm{Kcal} / \mathrm{mL})\end{array}$ \\
& PTN* $^{*}$ & LIP & CHO & \\
Fórmula com hidrolisado bovino & 14,3 & 20,4 & 65,3 & $0,59 \pm 0,02$ \\
Fórmula com hidrolisado de frango & 16,1 & 19,4 & 64,5 & $0,58 \pm 0,01$ \\
Fórmula com hidrolisado de peru & 17,0 & 15,9 & 67,0 & $0,58 \pm 0,03$ \\
\hline
\end{tabular}

* PTN - proteína; LIP - lipídeo; CHO - carboidrato

\section{Discussão}

Constatou-se a viabilidade de utilização de hidrolisado de carne como fonte protéica em fórmulas enterais artesanais, por fornecer proteína de alto valor biológico e parcialmente hidrolisada, o que facilita o processo absortivo.

A fonte de carne utilizada deve ser levada em consideração em casos de restrição do consumo de colesterol, sendo as fórmulas com carne de frango e peru as que apresentaram menor teor deste nutriente. O perfil de ácidos graxos observado nas fórmulas avaliadas é decorrente principalmente, dos lipídeos provenientes das carnes e do óleo vegetal utilizados, com prevalência dos monoinsaturados, seguido pelos saturados e com menor teor dos poliinsaturados.

Os minerais presentes nas fórmulas provém das carnes e dos legumes empregados, sendo a fórmula elaborada com carne bovina a que apresentou os maiores teores dos minerais estudados, conforme observado em tabelas de composição de alimentos. Verifica-se que a carne bovina, em relação ao frango e ao peru possui maior concentração de cobre, zinco e ferro.

As dietas avaliadas, classificadas como hipocalóricas, podem ser utilizadas na suplementação de pacientes que utilizam dietas enterais industrializadas ou caseiras ou ainda nos casos de uso concomitante dos dois tipos de fórmulas.

Além disso, estudos têm relatado o uso de formulações hipocalóricas em pacientes obesos que requerem nutrição enteral. DICKERSON et al, 2002, avaliou como positiva a utilização de nutrição enteral hipocalórica em pacientes obesos com obstrução intestinal ou perfuração, ferimentos a bala em cabeça e pescoço e agravos em geral de cabeça e pescoço que tinham a alimentação por via oral impossibilitada. Com a nutrição hipocalórica foi observada redução significativa de permanência na unidade de terapia intensiva e diminuição da duração da terapia com antibióticos, podendo ser orientada para uso no domicílio. 


\title{
5. Conclusão
}

A distribuição de nutrientes observada nas fórmulas deverá ser levada em consideração na sua aplicação, fazendo as correções necessárias, quanto à proporção de ingredientes e líquidos empregada frente a viscosidade e osmolalidade da fórmula.

As fórmulas não atingem $100 \%$ das recomendações de minerais, devendo-se avaliar a necessidade de suplementação, evitando-se possíveis déficits nutricionais.

Considerando-se o relatado aumento do uso de dietas enterais artesanais em terapia domiciliar, a determinação do teor de nutrientes das fórmulas utilizadas é de fundamental importância na adequada orientação dietética.

\begin{abstract}
This work aims to determine the centesimal composition of homemade enteral diets, based on meat protein hydrolysates. Methods: The obtention of the beef protein hydrolysates was as described by PINTO e SILVA et al, 1999 . Three formulae with meat hydrolysates (beef, chicken and turkey) were analyzed; they were added with rice, carrots, beetroot, corn starch, corn glucose and soybean oil. Centesimal composition were evaluated by the methodology recommended by INSTITUTO ADOLFO LUTZ, 1985. The cholesterol analysis was carried out by HPLC. The fatty acids were separated by high resolution gas chromatography; the values of food fibers according to the methodology presented by PROSKY et al, 1988. The minerals were determined by spectrophotometer of Atomic Absorption by equipment Perkin-Elmer model 5,100. Results: The formulae were classified as hypo caloric. The protein contents of the formulae varied from 2.09 to $2.52 \mathrm{~g} / 100 \mathrm{~g}$, lipids from 1.04 to $1.32 \mathrm{~g} / 100 \mathrm{~g}$, carbohydrates 9.32 and $9.67 \mathrm{~g} / 100 \mathrm{~g}$, containing $8 \mathrm{~g} / \mathrm{L}$ fibers. The cholesterol varied from $14.32 \mathrm{mg} / 100 \mathrm{~g}$ (chicken) to $37.59 \mathrm{mg} / 100 \mathrm{~g}$ (beef). As for fatty acids contents, it was verified that the three formulae show more concentration of monounsaturated fatty acids, prevailing the oleic. Concerning the minerals, the copper values varied from 0.07 $\mathrm{mg} / 100 \mathrm{~g}$ and $0.06 \mathrm{mg} / 100 \mathrm{~g}$, zinc from $0.28 \mathrm{mg} / 100 \mathrm{~g}$ to $0.62 \mathrm{mg} / 100 \mathrm{~g}$, iron from $0.47 \mathrm{mg} / 100 \mathrm{~g}$ to $0.63 \mathrm{mg} / 100 \mathrm{~g}$, magnesium from $8.08 \mathrm{mg} / 100 \mathrm{~g}$ to $8.52 \mathrm{mg} / 100 \mathrm{~g}$ and calcium from $1.34 \mathrm{mg} / 100 \mathrm{~g}$ to $1.40 \mathrm{mg} / 100 \mathrm{~g}$. The formulae presented adequate protein contents, predominance of monounsaturated fatty acids, being recommended to patients who require nutritional supplementation.
\end{abstract}

Key words: enteral nutrition, centesimal composition, minerals.

\section{Referências}

ARAÚJO, E.M.; MENEZES, H.C. Composição centesimal, lisina disponível e digestibilidade in vitro de proteínas de fórmulas para nutrição oral ou enteral. Cienc. Tecnol. Aliment., n. 4, v. 25, p. 768-771, 2005.

ATZINGEN, M.C.V. Desenvolvimento de dieta enteral artesanal com hidrolisado protéico de carne e determinação de parâmetros químicos e nutricionais [dissertação]: Universidade de São Paulo, 2005.

BAXTER, Y.C.; WAITZBERG, D.L.; PINOTTI, H.W.; RODRIGUES, J.G. Fórmulas enterais poliméricas e especializadas. In: WAITZBERG DL Nutrição oral, enteral e parenteral na prática clínica. $3^{\mathrm{a} e d . ~ S a ̃ o ~ P a u l o: ~}$ Atheneu, 2001. p. 677-695. 
BOHAC, C.E.; RHEE, L.S.; CROSS, H.R.; ONO, K. Assesment of methodologies for colorimetric cholesterol assay of meats. J. Food Sci., v. 53, p. 1642-1644,1988.

CAMPBELL, J.M.; FAHEY, J.R.G.C.; LICHTENSTEIGER, C.A.; DEMICHELE, S.J.; GARLEB, K.A. An enteral formula containing fish oil, indigestible oligosaccharides, gum arabic and antioxidants affects plasma and colonic phospholipid fatty acid and prostaglandin profiles in pigs. J. Nutr., v.127, p. 137-145, 1997.

CHEN, B.H.; CHEN, Y.C. Evaluation of the analysis of cholesterol oxides by liquid chomatography. J. Chromatogr. A., v. 661, p. 127-136, 1994.

DICKERSON, R.N.; BOSCHERT, K.J.; KUDSK, K.A.; BROWN, R.O. Hypocaloric enteral tube feeding in critically ill obese patients. Nutr., v.18, p. 241-246, 2002.

DOMENE, S.M.A.; GALEAZZI, M.A.M. Prescrição e uso de formulados para nutrição enteral pelos serviços de nutrição hospitalares do município de Campinas - São Paulo, Brasil. Rev Nutr PUCCAMP, n. 2, v. 10, p. 114-119, 1997.

EPLEY, D. Nutritional assessment in home care patients. Home Care Prov., n. 3, v. 4, p. 102-105, 1999.

HEBUTERNE, X.; BOZZETTI, F.; MORENO VILLARES, J.M.; PERTKIEWICZ, M.; SHAFFER, J.; STAUN, M.; et al. Home enteral nutrition in adults: a European multicentre survey. Clin. Nutr., n. 3, v. 22, p. 261-266, 2003.

INSTITUTE OF MEDICINE. Dietary Reference Intake: applications in dietary assessment. Washington, 2000.

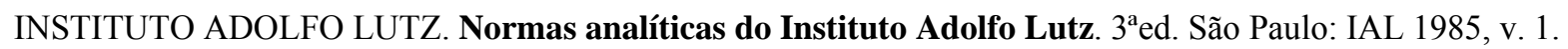

MARKS, G.E.; MOORE, C.E.; KANABROCKI, E.L.; OESTER, Y.T.; KAPLAN, E. Determination of trace elements in human tissue. I, Cd, Fe, Zn, Mg and Ca. Appl. Spectrosc., v. 26, 1971.

MARMER, W.N.; MAXWELL, R.J. Dry columm method for the quantitative extraction and simultaneous class separation of lipids from muscle tissue. Lipids, v.16, p. 365-371, 1981.

MITNE, C.; SIMÕES, A.M.G.; WAKAMOTO, D.; LIORI, G.P.; SULliVAN, M.; COMER, G.M. Análise das dietas enterais artesanais. Rev. Bras. Nutr. Clin., v. 16, p. 100-109, 2001.

MONTEJO, O.; ALBA, G.; CARDONA, D.; ESTELRICH, J.; MANGUES, M.A. Relación entre la viscosidad de las dietas enterales y las complicaciones mecânicas en su administración según el diâmetro de la sonda nasogástrica. Nutr. Hosp., n.2, v.16, p. 41-45, 2001.

MORAIS, T.B.; VIEIRA, M.M.C. Qualidade nutricional de dietas enterais preparadas em residências de pacientes em terapia nutricional domiciliar, na região metropolitana de São Paulo - SP. Rev. Bras. Nutr. Clin., n. S2, v. 20, p. 16, 2005 .

MORRISON, W.R.; SMITH, L.M. Preparation of fatty acids methyl esters and dimethylacetals from lipids with boron fluoride-methanol. J. Lip. Res., v. 5, p. 600-608, 1964.

NEPA - UNICAMP. Tabela brasileira de composição de alimentos, disponível em <http: www.unicamp.br/nepa/taco $>$, acesso em 10/04/2007.

NISIM, A.A.; ALLINS, A.D. Enteral nutrition support. Nutr., v. 21, p. 109-112, 2005.

PINTO e SILVA, M.E.M; MAZZILLI, R.N.; CUSIN, F. Composition of hydrolysates from meat. J. Food Comp. Anal., v. 12, p. 219-225, 1999.

PREETIKA, A,; PADMINI, G.; SHOBHA, U. Nutrient dense mixes for enteral feeding in India. Nutr. Food Sci., v. 34 , p. 277-281, 2004.

PROSKY, L. ASP.; SCHWEIZER, T.F.; DEVRIES, J.W.; FURDA, I. Determination of insoluble, soluble and total dietary fiber in foods and food products: interlaboratory study. J. Assoc. Off. Anal. Chem., n. 5, v. 71, p. 1017-1023, 1988. 
SILVA, J.W. Estudo comparativo das complicações da gastrostomia e jejunostomia em pacientes do serviço de cabeça e pescoço [monografia]: Universidade Federal do Paraná, 2002.

SULLIVAN, M.M.; SORREDA-ESGUERRA, P.; SANTOS, E.E.; PLATON, B.G.; CASTRO, C.G.; IDRISALMAN, E.R.; et al. Bacterial contamination of blendereized whole food and comercial enteral tube feedings in the Phillippines.

J. Hosp. Inf., v. 49, p. 263-273, 2001.

VICENTE, S.J.V. Formação de óxidos de colesterol em hambúrguer bovino em função do binômio tempo/temperatura de preparação [dissertação]: Universidade de São Paulo, 2003.

YAMAUTI, A.C.; ANDRADE, K.C.; CORDELINI, S.; PIASSA, H.H.C.S. Nutrição em home care: importância da adaptação dietética à realidade domiciliar. Rev. Bras. Home Care, v. 51, p. 4-5,1999.

Primeiro autor:

Nome completo: Maria Carolina Von Atzingen

Filiação institucional: Faculdade de Saúde Pública - USP - São Paulo - Brasil

Departamento: Nutrição

Função ou cargo ocupado: doutorando

Endereço completo para correspondência (bairro, cidade, estado, país e CEP): Av. Dr. Arnaldo, 715, Cerqueira César, São Paulo - SP - BRASIL, cep. 01246904.

Telefones para contato: 1130617771 r 226 / 1130617701 r 226

e-mail:mcva@usp.br

Nome completo: Maria Lima Garbelotti

Filiação institucional: Instituto Adolfo Lutz

Departamento: Alimentos

Função ou cargo ocupado: Química

Endereço completo para correspondência (bairro, cidade, estado, país e CEP): Av. Dr. Arnaldo, 355 Cerqueira César, São Paulo - SP - BRASIL, cep. 01246902

Telefones para contato: 30682938

e-mail: mgarbelo@ial.sp.gov.br

Nome completo: Roberta Faria Camilo Araújo

Filiação institucional: Universidade Federal de São Paulo

Departamento: Bromatologia

Função ou cargo ocupado: Técnico de Laboratório

Endereço completo para correspondência (bairro, cidade, estado, país e CEP): Rua Botucatu, 740, cep. 04023900

Telefones para contato: 55764000

e-mail: omsamancio.dped@epm.br

Nome completo: Rosana Manólio Soares 
Filiação institucional: Faculdade de Saúde Pública - USP - São Paulo - Brasil

Departamento: Nutrição

Função ou cargo ocupado: Técnico de Laboratório

Endereço completo para correspondência (bairro, cidade, estado, país e CEP): Av. Dr. Arnaldo, 715, Cerqueira César, São Paulo - SP - BRASIL, cep. 01246904.

Telefones para contato: 1130617765

e-mail: rosanaso@usp.br

Nome completo: Maria Elisabeth Machado Pinto e Silva

Filiação institucional: Faculdade de Saúde Pública - USP - São Paulo - Brasil

Departamento: Nutrição

Função ou cargo ocupado: Docente

Endereço completo para correspondência (bairro, cidade, estado, país e CEP): Av. Dr. Arnaldo, 715, Cerqueira César, São Paulo - SP - BRASIL, cep. 01246904.

Telefones para contato: 1130617771 r 226 / 1130617701 r 226

e-mail:mmachado@usp.br 